\title{
Overall brightness decrease observed in the Ehrenstein illusion induced for both contrast polarities
}

\author{
JIRO HAMADA \\ Tokushima University, Tokushima, Japan
}

\begin{abstract}
Brightness of both the area at the center of the Ehrenstein illusion and the area outside the center was measured for figures of both contrast polarities by matching them to Munsell gray patches (reference Munsell values $>6$ ). Relative illusory enhancement of brightness or darkness was observed at the center of the Ehrenstein figures with dark or light lines, respectively. However, the absolute brightness measured at the center and outside the center of the Ehrenstein figures revealed an overall darkness enhancement for both contrast polarities. A three-stage hypothetical model is proposed to explain the results qualitatively: (1) The light and dark illusory patches may be due to the possibility that activity at the receptive-field center region spreads laterally and angle-dependently into the background, whereas no such spreading occurs within the central gap between radial lines. (2) Subjective contours may result from the boundary of the lateral spread into the background. (3) The overall brightness decrease observed in Ehrenstein figures of both contrast polarities may be due to an additional spreading of nonantagonistic inhibition.
\end{abstract}

In the Ehrenstein (1941) illusion, a light patch is perceived between the ends of dark radial lines on a light background (negative contrast) and a dark patch is perceived between the ends of light radial lines on a dark background (positive contrast). Spillmann, Fuld, and Gerrits (1976) and Spillmann, Fuld, and Neumeyer (1984) reported, on the basis of threshold and suprathreshold measurements, that the illusion was stronger with positive contrast. Fuld and O'Donnell (1984) confirmed, for an Ehrenstein figure of negative contrast, Ehrenstein's qualitative observation that increasing the gap between the ends of the radial lines results in increased brightness enhancement. It is difficult to explain these findings in terms of classical simultaneous contrast based on lateral inhibition. Day and Jory $(1978,1980)$ therefore introduced use of the term "line contrast" in addition to simultaneous contrast. They assumed that the line contrast, present in the Ehrenstein illusion, was the result of assimilative and dissimilative processes.

Spillmann (1985) reviewed the peculiarities of the Ehrenstein illusion and related phenomena as clarified by phenomenological, psychophysical, and neurophysiological approaches. Up to now, the amount of assimilation occurring in the background of an Ehrenstein pattern has been described only qualitatively (Ehrenstein, 1941; Day \& Jory, 1978; it has not yet been investigated quantitatively. The present study investigated the following ques-

This research was supported by Grants-in-Aid for Scientific Research (59710073, 60510066) from the Japanese Ministry of Education, Science and Culture. L. Spillmann, W. H. Ehrenstein, T. S. Aiba, M. Akita, and M. J. Morgan improved the manuscript. S. Grossberg, Y. Ejima, K. Amano, and N. Osaka provided helpful comments. M. Suezaki helped in collecting data. Their assistance is gratefully acknowledged.

The author's mailing address is: Department of Behavioral Science, Tokushima University, Tokushima 770 , Japan. tions: (1) What are the brightnesses of the Ehrenstein figure as measured in the central gap between the radial line ends and on the background between the lines lying side by side? (2) How does the strength of the illusion depend on the contrast polarity? (3) What is the effect of the gap diameter?

\section{METHOD}

\section{Apparatus and Stimuli}

Figure 1 shows the side (a) and front (b) views of the experimental apparatus. Eight quartz-iodine lamps $(6 \mathrm{~V}, 20 \mathrm{~W})$ illuminated a light-gray circular screen (Munsell N7.0, $6^{\circ} 10^{\prime}$ of visual angle) and its black surround area (Munsell N2.5, $38^{\circ}$ of visual angle, both in width and height). When illuminated in this manner, the luminance of the round screen was $19 \mathrm{~mL}$, and the luminance of the square surround area was $2 \mathrm{~mL}$. Six stimulus fields were employed: four different Ehrenstein figures and two different round uniform control fields. A slide projector $(100 \mathrm{~V}, 250 \mathrm{~W})$ was used to superimpose the stimulus fields onto the screen. They were presented for a duration of $2 \mathrm{sec}$. Figure 2 illustrates the stimulus fields used. Each Ehrenstein figure consisted of eight lines, dark on a light background (negative contrast) or light on a dark background (positive contrast). The luminances of the light lines and the background in negative contrast were $44 \mathrm{~mL}$, and those of the dark lines and the background in positive contrast were $19 \mathrm{~mL}$. The lines of the Ehrenstein figures subtended $2^{\circ} 36^{\prime}$ or $2^{\circ} 05^{\prime}$ in length and $3^{\prime}$ in width. The outer diameter of the entire figure was $5^{\circ} 43^{\prime}$. Two gap diameters of either $31^{\prime}$ or $1^{\circ} 33^{\prime}$ were used. The small gap diameter of $31^{\prime}$ was similar to the largest one used by Fuld and O'Donnell (1984). Two uniform fields with the same luminances ( 44 and $19 \mathrm{~mL}$ ) and the same diameter as the backgrounds of the two Ehrenstein figures served as controls. To measure the apparent brightnesses of the central gap and the background of the Ehrenstein figure, and of the uniform control field, 33 Munsell patches of various shades of gray were used as comparison stimuli. They were presented in a comparison window ( $30^{\prime}$ wide and $1^{\circ} 45^{\prime}$ high), located $5^{\circ} 43^{\prime}$ of arc above the round screen. The gray values ranged 
SIDE VIEW

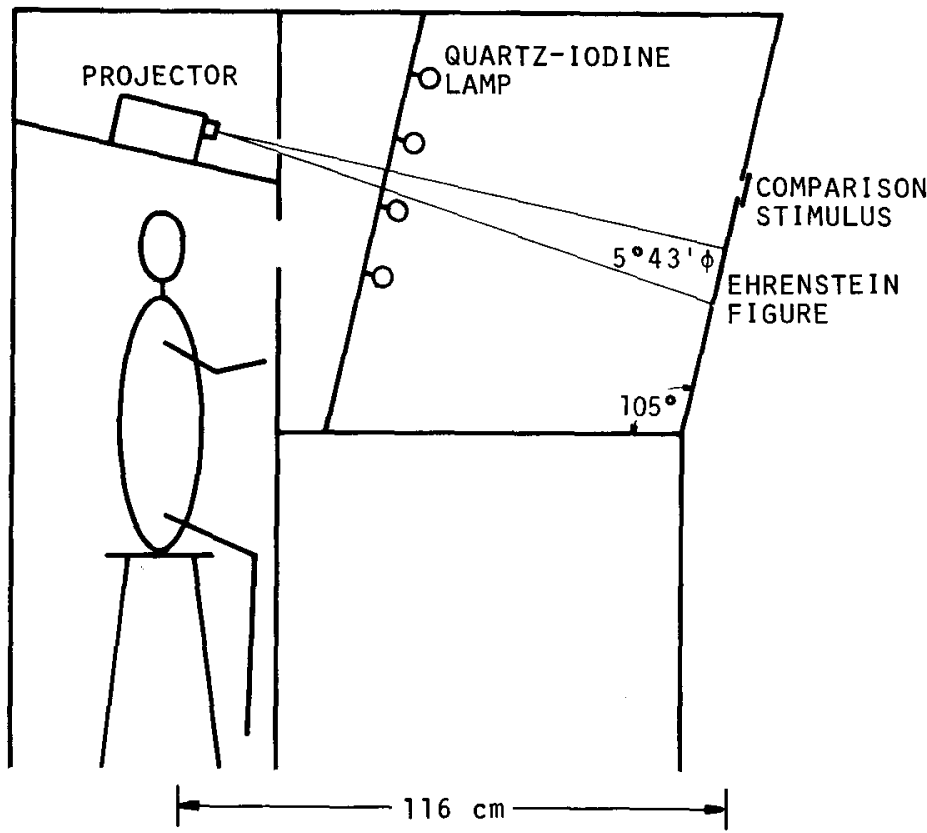

(a)
FRONT VIEW

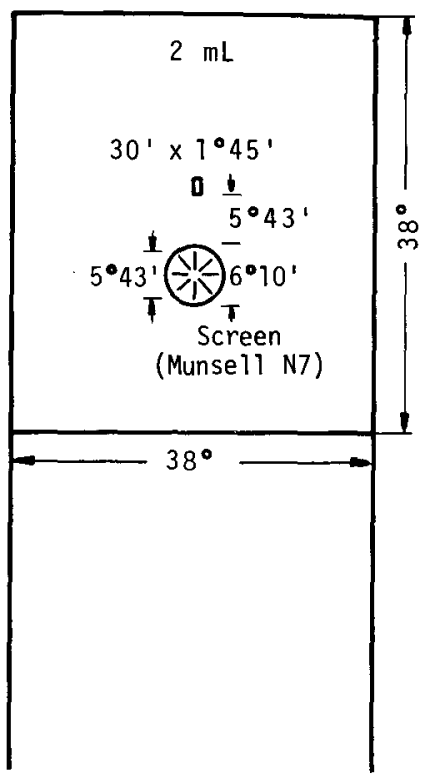

(b)

Figure 1. Schematic representation of the experimental apparatus. For further details, see Method section.

from Munsell N1.5 to N9.5 in steps of N0.25; the corresponding luminances varied from 0.88 to $40 \mathrm{~mL}$.

\section{Procedure}

Five subjects (4 students in an undergraduate course and the author) participated in the experiment. All observers had normal or corrected-to-normal vision. The head was stabilized by a headand chinrest. The distance between the subject and the circular screen was $116 \mathrm{~cm}$. The task of the subject was to compare the apparent brightnesses of both the illusory patch and the background in the Ehrenstein figure, as well as of the uniform field, with those of the Munsell gray patches shown in the rectangular window above. Three categories to judge the apparent brightnesses were used: "darker," "lighter," or "equal." The method of limits was used to obtain a point of subjective equality, which was the mean of two upper limits and two lower ones. They were measured by both an ascending series and a descending series in an experimental session, and were defined by a transition of the three categories. The sequences of stimulus presentation were counterbalanced. During the task, each subject moved his or her eyes freely between the test and the comparison stimuli. The subjects made all comparisons foveally by looking back and forth. The subjects started with a practice session and then proceeded to six experimental sessions, each on a different day.

\section{RESULTS}

The means and $1 S D$ from 5 subjects are shown in Figure 3 as a function of the gap diameter with contrast polarity as parameter. The brightness of the illusory light patch (filled circles, ๑; about Munsell N6.9) in negative contrast figures was darker than that of the correspond-

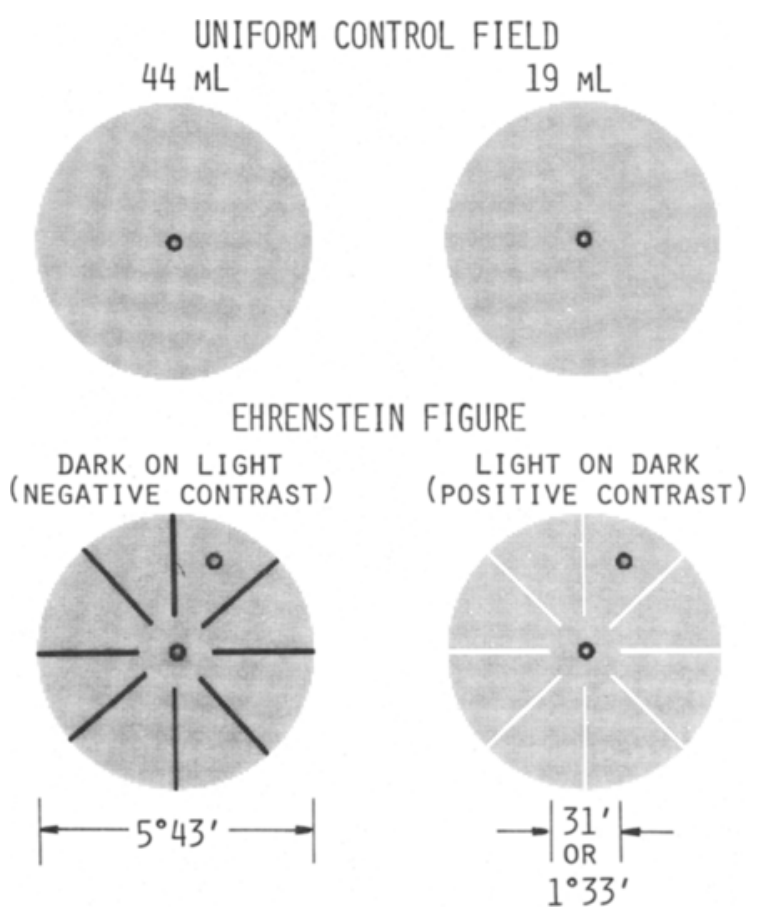

Figure 2. Illustrations of test stimuli. Black/white lines on the screen-tone refer to dark/light lines on light/dark background in the Ehrenstein figure. Subjects quantified the apparent brightnesses of the positions indicated by the open circles $(0)$ by comparison with Munsell gray papers. 


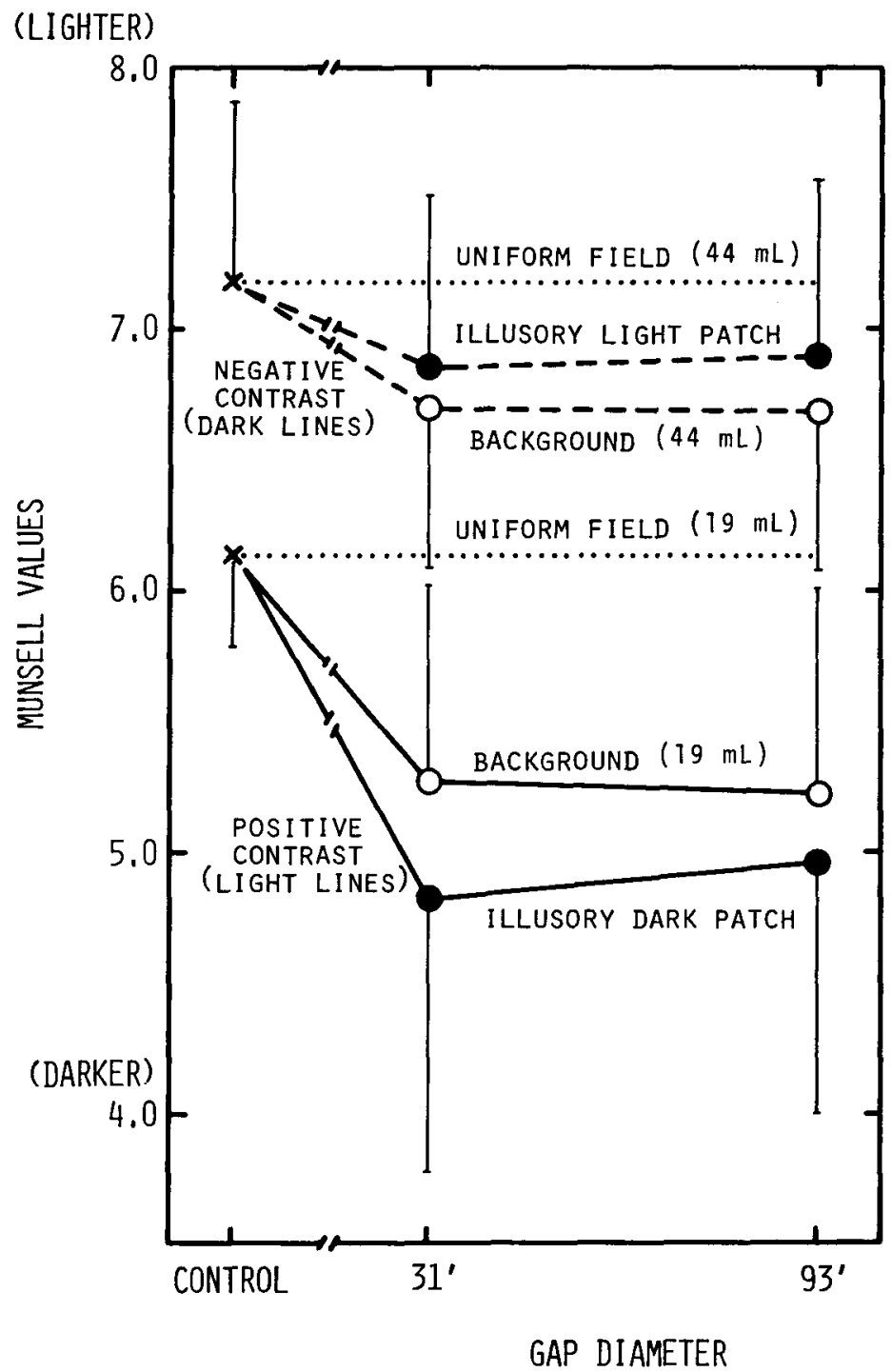

Figure 3. Average Munsell gray values from 5 subjects as a function of gap diameter of the Ehrenstein figure with contrast polarity as parameter (broken and solid lines indicate negative and positive contrasts). Brightnesses of the illusory patch (filled circle, $\bullet$ ), the background (open circle, o), and uniform field (cross, $x$ ) are depicted. Dotted lines indicate the brightnesses of the uniform control fields. The vertical bars on the data points indicate $1 S D$ of 5 subjects.

ing uniform field (cross, $x$; N7.2). A two-way analysis of variance involving two figures, 5 subjects, and six sessions yields significant differences for the main effect of figures, that is, the uniform control field and the illusory light patch for both the small gap diameter $[F(1,50)=$ $6.74, p<.05]$ and the large one $[F(1,50)=4.37$, $p<.05]$. The main effect of subjects was highly significant $\left[F_{\mathrm{s}}(4,50)=31.7\right.$ and $\left.30.0, p<.01\right]$, whereas the figures $\times$ subjects interaction was not significant $\left[F_{\mathrm{s}}(4,50)\right.$ $=0.56$ and $0.76, p>.05$ ]. The brightnesses of both the light backgrounds (open circles, $0 ;$ N5.3) and the dark patches (filled circles, $\bullet$; N4.9) in the positive contrast figures were clearly below those of the corresponding uniform fields (cross, $X$; N6.1).

With an increase of gap diameter, the brightnesses of the two locations, that is, the central gap and the background, of the figures in both contrast polarities remained constant. For the negative figure-ground contrast, a threeway analysis of variance on the locations (2) $\times$ gap diameters (2) $\times$ subjects (5) with six sessions revealed that the main effects of locations $[F(1,100)=5.39, p<.05]$ and of subjects $[F(4,100)=65.0, p<.01]$ achieved significance, whereas that of gap diameters $[F(1,100)=$ $0.06, p>.05$ ] and all of the interactions did not yield 
significant differences. For the figure with a positive contrast polarity, the analysis of variance also yielded significant differences between two locations $[F(1,100)=$ $22.5, p<.01]$ and among 5 subjects $[F(4,100)=138.8$, $p<.01]$, but not between two gap diameters $[F(1,100)$ $=0.34, p>.05]$. The locations $\times$ subjects interaction was found to be significant $[F(4,100)=3.26, p<.05]$, but none of the other interactions were.

The average data from two figures with the small and large gap diameters indicated that the brightness differences between the illusory patches and the backgrounds were represented by Munsell N0.4 and N0.2 for positive and negative contrast, respectively. The three-way analysis of variance on the contrast polarities (2) $\times$ gap diameters $(2) \times$ subjects $(5)$ with six sessions yielded significant differences between the contrast polarities $[F(1,100)=15.1, p<.01]$ and among the 5 subjects $[F(4,100)=15.5, p<.01]$, but not between the two gap diameters $[F(1,100)=2.79, p>.05]$. Thus, the Ehrenstein illusions were greater for the positive contrast figures than for the negative contrast ones.

\section{DISCUSSION}

The data plotted in Figure 3 indicate that (1) the light and dark illusory patches were observed in the Ehrenstein figures of negative and positive contrasts, and (2) regardless of negative and positive contrast, the brightnesses of both the illusory patch and the background of the Ehrenstein figures were darker than the brightness of the uniform control field. The results may qualitatively be explained by a three-stage model developed by Hamada (1984a, 1984b, 1985). The model assumes antagonistic and nonantagonistic mechanisms at early stages of the visual system and the reconstructive process of a lateral spread at a higher stage. The antagonistic mechanism corresponds to the lateral inhibition of neural units (Békésy, 1968) and their psychophysical counterparts, that is, perceptive fields (Jung \& Spillmann, 1970). In addition, to explain the brightness decrease in both the light and dark stripes of square-wave gratings and the Craik-O'Brien pattern, which could not be explained by antagonistic inhibition, nonantagonistic inhibition (Hamada 1984a, 1985) is assumed to diffuse widely over a large area in a pattern. A study of Freund, Hennerici, and Rabenschlag (1977) indicates that synergistic reversal of surround- into center-type responses might be involved as a neural basis for nonantagonistic inhibition. Furthermore, Hamada (1985) applied Gerrits and Vendrik's (1970) concept of the lateral spreading of center-surround activities to explain the Craik-O'Brien illusion. The lateral spreading of neural activity is assumed to be halted by a barrier that corresponds to the position of the inflection point or the zero-crossing (Hamada, 1984b; Marr \& Hildreth, 1980; Watt \& Morgan, 1983) of the neural profile. The Ehrenstein illusion can be explained by a three-stage model (see Figure 4) as follows.
Excitation/inhibition organized at a first stage, for dark and light lines on uniform fields, is shown in Figures 4a and $\mathrm{a}^{\prime}$, respectively. A dark line reduces excitation at the line and reduces inhibition around it (Figure 4a), whereas a light line induces excitation at the line and induces inhibition around it (Figure 4a'). The neural profile for each line in antagonistic processing may be approximated by the difference of two Gaussians (DOG), which correspond to center and surround fields. A computer simulation on border contrast (Hamada, 1984b) showed that the ratio of the center and surround space constants of DOG filters was about 9.0, and that the ratio of the strengths of center and surround activities was about 0.19. Thus, total response at the center should be stronger than that at the surround. Nonantagonistic inhibition, which is produced at a second stage, suppresses the excitation induced by the dark line, whereas the same inhibition enhances the antagonistic inhibition induced by the light line, as shown in Figures $4 b$ and $b^{\prime}$. Thus, inhibition results in the overall darkening seen in Ehrenstein figures of either negative or positive contrast. Nonantagonistic inhibition should be stronger than antagonistic inhibition, because the brightness of illusory light patch in the figure of negative contrast was darker than that of the uniform control field. The neural profile resulting from both the antagonistic and nonantagonistic mechanisms should have two inflection points around the dark/light lines, as indicated by the open circles in Figures $4 b$ and $b^{\prime}$. In the two-dimensional space, the distribution of neural activity for the line has a contour line that corresponds to inflection points. Lastly, the neural profile compounded by the antagonistic and nonantagonistic processes is further processed at a third stage in which lateral spreading takes place. It is assumed here that neural activities inside the subjective contour line should be homogenized and those outside it (hatched areas in Figures $4 b$ and $b^{\prime}$ ) should spread to the right angle against the dark/light lines, as shown in Figures $4 c$ and $c^{\prime}$. Then angle-dependent lateral spreading might lead to the illusory light and dark patches perceived in the Ehrenstein figure, since the background receives lateral spreading, whereas the central gap does not. The resulting neural activities are schematically indicated by light and dark screen-tone sections in Figures $4 d$ and $d^{\prime}$, where the boundary of the lateral spread in the Ehrenstein illusion is shown by a circular subjective contour. The lateral spreading might correspond to assimilation on both sides of a line (Helson, 1963), whereas the lack of spreading within the gap of the line ends might cause dissimilation (Day \& Jory, 1978, 1980).

Hamada (1985) found an asymmetry of the Craik$O$ 'Brien illusion, in which a pattern of negative contrast induced a stronger illusion than did that of positive contrast. In contrast to the Craik-O'Brien illusion, positive contrast was stronger than negative contrast in the Ehrenstein illusion, consistent with other results (Spillmann, Fuld, \& Gerrits, 1976; Spillmann, Fuld, \& Neumeyer, 1984). The reverse asymmetry in strength in the Craik- 


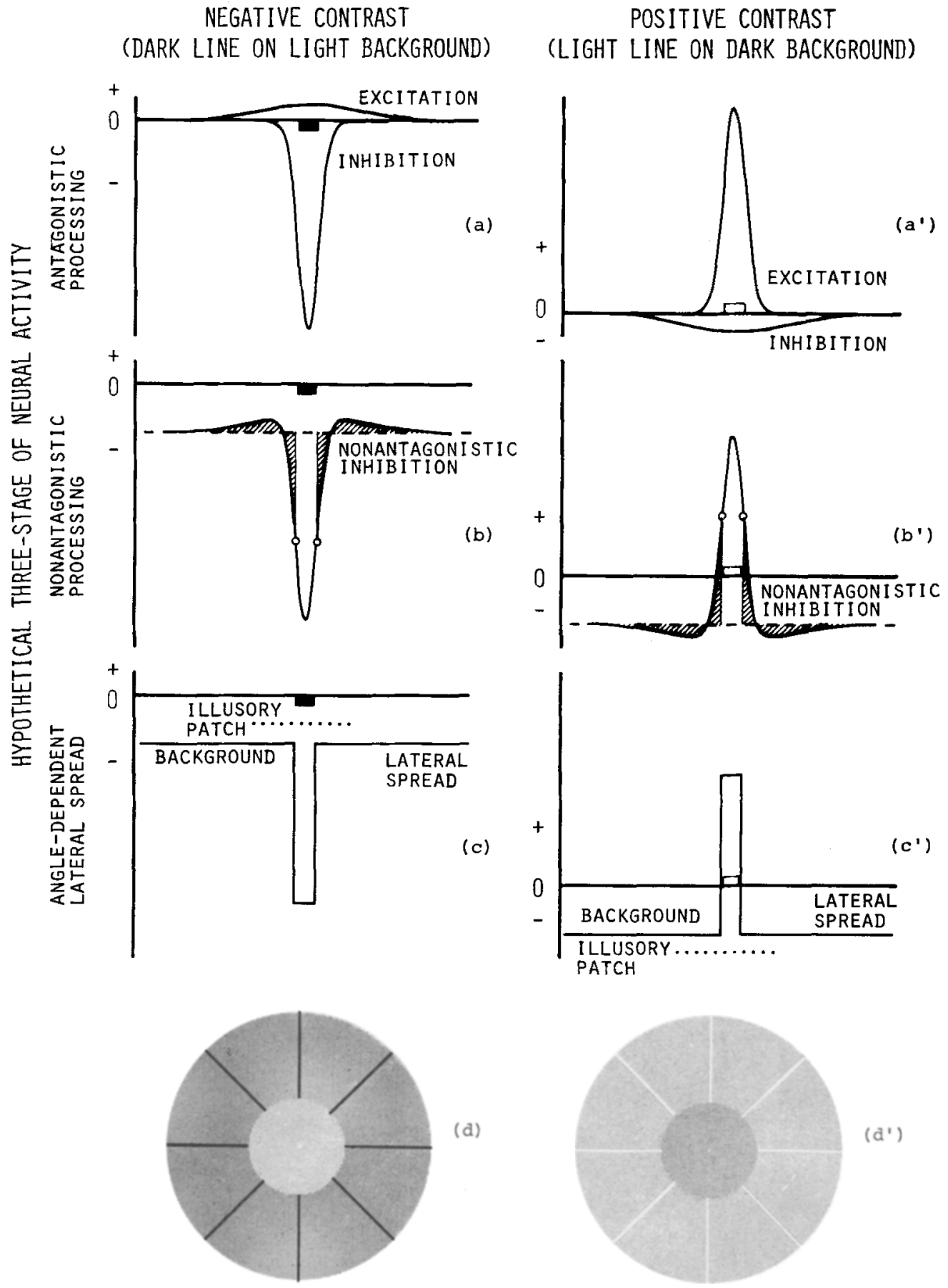

Figure 4. Three-stage model of hypothetical neural activities generating the Ehrenstein illusion. Cases of negative figure-ground contrast are shown on the left; those of positive contrast are shown on the right. The value, 0 , on the ordinate indicates the neural activity corresponding to the uniform control field. The broken lines in (b) and (b') indicate the activity level inhibited nonantagonistically in the Ehrenstein figure. The dotted lines in (c) and (c') indicate the neural activity at the central gaps between the ends of the radial lines. The brightnesses for the Ehrenstein figure are schematically indicated by light and dark screen tone in (d) and (d'). The center-surround fields organized by antagonistic excitation/inhibition at the first stage are shown in (a) and (a'). At a second stage, the nonantagonistic inhibition is widely diffused, corresponding to an overall darkening, as shown in (b) and (b'). These neural activities are assumed to be produced at early levels of the visual system, and to be sent to a higher level. At a third stage, lateral spreading, which is halted at locations of inflection point indicated by open circles in (b) and (b'), takes place. A subjective contour line corresponds roughly to the infection points around the line in two-dimensional spoce. The neural activities inside the contour line are homogenized, and those outside the line (hatched area) spread to right angle against the line, as shown in (c) and (c'). The light and the dark illusory patches produced are shown in (d) and (d'). An explanation for this is as follows: Angle-dependent lateral spreading takes place only outside the subjective contour line (background), but not in the central gap. Thus, a semicircular subjective contour appears at the boundary of lateral spreading. 
O'Brien and Ehrenstein illusions found for both contrast polarities should be investigated in further studies.

Cohen and Grossberg (1984) and Grossberg and Mingolla (1985) recently distinguished two parallel contoursensitive processes (boundary contour and feature contour processes), and correctly predicted the brightness paradoxes reported by Bergström (1966) and Hamada $(1982,1985)$. The boundary contour process is supposed to be sensitive to the orientation and amount of contrast in edges, and the feature contour process is sensitive to both the amount and direction of contrast. The orientationdependent boundary grouping process of Grossberg's model generates the contours. In addition, his model proposes a nonoriented filling-in process. On the other hand, the model presented here assumes that the inflection points are extracted as edges or contours which halt the angledependent lateral spreading at a higher stage of the human visual system.

\section{Conclusion}

This study shows that when the light or dark illusory patches are observed in the Ehrenstein figure with dark or light lines, the brightnesses both of the illusory patch and of the background are darker than that of the uniform control field. The results can be explained by the threestage model as follows: (1) The illusory light and dark patches are not due to the summation of activities at the antagonistic receptive-field surround region, but are due to the angle-dependent lateral spreading of the center activities at the field; (2) the semicircular subjective contour is produced by the boundary of the angle-dependent lateral spread; and (3) the overall brightness decrease in the Ehrenstein illusion of both contrast polarities may be due to additional spreading of nonantagonistic inhibition (Hamada, 1982, 1985).

\section{REFERENCES}

BÉKÉSY, G. voN (1968). Mach- and Hering-type lateral inhibition in vision. Vision Research, 8, 1483-1499.

BergSTröm, S. S. (1966). A paradox in the perception of luminance gradients. I. Scandinavian Journal of Psychology, 7, 209-224.

Cohen, M. A., \& Grossberg, S. (1984). Neural dynamics of brightness perception: Features, boundaries, diffusion, and resonance. Perception \& Psychophysics, 36, 428-456.

DAY, R. H., \& JoRY, M. K. (1978). Subjective contours, visual acuity, and line contrast. In J. C. Armington, J. Krauskopf, \& B. R. Wooten
(Eds.), Visual psychophysics and physiology (pp. 331-340). New York, San Francisco, London: Academic Press.

DAY, R. H., \& JORY, M. K. (1980). A note on a second stage in the formation of illusory contours. Perception \& Psychophysics, 27, 89-91.

EhrensteIn, W. (1941). Über Abwandlungen der L. Hermannschen Helligkeitserscheinung. Zeitschrifi für Psychologie, 150, 83-91.

Freund, H.-J., Hennerici, M., \& Rabenschlag, U. (1977). Reversal of surround- into center-type responses of cat retinal ganglion cells by local darkening of the receptive field center. Vision Research, 17, 487-494.

FulD, K., \& O'Donnell, K. (1984). Brightness matching and scaling of the Ehrenstein illusion. In L. Spillmann \& B. R. Wooten (Eds.), Sensory experience, adaptation, and perception (pp. 461-469). Hillsdale, NJ: Erlbaum.

GerRITs, H. J. M., \& Vendrik, A. J. H. (1970). Simultaneous contrast: Filling-in process and information processing in man's visual system. Experimental Brain Research, 11, 411-430.

Grossberg, S., \&ingolla, E. (1985). Neural dynamics of form perception: Boundary completion, illusory figure, and neon color spreading. Psychological Review, 92, 173-211.

HAmadA, J. (1982). The contour enhancement effects produced by darkening effects. In H.-G. Geissler \& P. Petzold (Eds.), Psychophysical judgment and the process of perception (pp. 132-139). Amsterdam: North-Holland.

Hamada, J. (1984a). Lightness decrease and increase in square-wave gratings. Perception \& Psychophysics, 35, 16-21.

Hamada, J. (1984b). A multi-stage model for border contrast. Biological Cybernetics, 51, 65-70.

Hamada, J. (1985). Asymmetric lightness cancellation in Craik-O'Brien patterns of negative and positive contrast. Biological Cynbernetics, 52, $117-122$.

HeLson, H. (1963). Studies of anomalous contrast and assimilation. Journal of the Optical Society of America, 53, 179-184.

JUNG, R., \& SILlmANN, L. (1970). Receptive-field estimation and perceptual integration in human vision. In F. A. Young \& D. B. Lindsley (Eds.), Early experience and visual information processing in perceptual and reading disorders (pp. 181-197). Washington, DC: National Academy of Sciences.

Marr, D., \& HiLdReth, E. (1980). A theory of edge detection. Proceedings of the Royal Society of London (B), 207, 187-217.

Spillmann, L. (1985). Illusory brightness and contour perception: Current status and unresolved problems. Manuscript submitted for publication.

Spillmann, L., Fuld, K., \& Gerrits, H. J. M. (1976). Brightness contrast in the Ehrenstein illusion. Vision Research, 16, 713-719.

SpillmanN, L., Fuld, K., Neumeyer, C. (1984). Brightness matching, brightness cancellation, and increment threshold in the Ehrenstein illusion. Perception, 13, 513-520.

WatT, R. J., \& Morgan, M. J. (1983). Mechanisms responsible for the assessment of visual location: Theory and evidence. Vision Research, 23, 97-109.

(Manuscript received April 14, 1986; revision accepted for publication October 8, 1986.) 